\title{
Restabelecimento da estética do sorriso em paciente com hipoplasia de esmalte
}

Esthetic smile rehabilitation of patient with enamel hypoplasia

Restablecimiento de la estética de la sonrisa en paciente con hipoplasia de esmalte

Kalyne Izael ROCHA ${ }^{1}$

Lilianne Nunes TELES ${ }^{1}$

Diala Aretha de Sousa FEITOSA ${ }^{2}$

${ }^{1}$ Acadêmico (a) do Curso de Odontologia do Centro Universitário Doutor Leāo Sampaio, UNILEÃO, 63040-405 Juazeiro do Norte - CE, Brasil

${ }^{2}$ Professora Doutora em Dentistica, Centro Universitário Doutor Leão Sampaio, UNILEÃO, 63040-405 Juazeiro do Norte - CE, Brasil

\section{Resumo}

A hipoplasia de esmalte pode ser entendida como uma formação incompleta ou defeituosa do esmalte dentário, apresentando desde um manchamento superficial de aspecto esbranquiçado a alterações severas de cor e textura. $\mathrm{O}$ esmalte pode mostrar-se irregular, rugoso, amolecido e com cavitação. A profundidade e causa dessas manchas é o fator chave na seleção e sucesso do tratamento. O objetivo do presente estudo é relatar um caso de reestabelecimento da estética do sorriso em um paciente com hipoplasia de esmalte, através da associação de tratamentos minimamente invasivos. Para o caso, optou-se pela associação técnica de microabrasão do esmalte dental com ácido fosfórico $37 \%$ associado à pedra-pomes e a macroabrasão do esmalte. Ao final do presente caso, foi devolvida de forma satisfatória a harmonia do sorriso do paciente de acordo com suas expectativas. Quando bem indicada, a associação de tratamentos minimamente invasivos para remoção de manchas decorrentes da hipoplasia do esmalte demonstra sucesso.

Descritores: Anormalidades Dentárias; Esmalte Dentário; Estética Dentária; Microabrasão do Esmalte; Resinas Compostas.

\section{Abstract}

Enamel hypoplasia is a defect characterized by an incomplete or insufficient formation of dental enamel. This condition can take many forms, ranging from a superficial whitish stain to severe changes in color and texture. The enamel can be irregular, rough, soften and eroded. The main cause and depth of the enamel stains is the key factor for a successful treatment. This study aimed to report a case of aesthetic smile rehabilitation performed in a patient with enamel hypoplasia by the association of minimally invasive treatment. Dental enamel microabrasion with phosphoric acid at $37 \%$ and pumice was associated with enamel macro abrasion technique. As a result, the smile harmony achieved by the techniques used was satisfactory and met the patient's expectations. The association between minimally invasive techniques showed good results when performed in enamel hypoplasia stains.

Descriptors: Tooth Abnormalities; Dental Enamel; Esthetics, Dental; Enamel Microabrasion; Composite Resins.

\section{Resumen}

La hipoplasia de esmalte puede ser entendida como una formación incompleta o defectuosa del esmalte dental. En el cual se presenta desde un manchamiento superficial de aspecto blanquecino, pudiendo llegar incluso a alteraciones severas de color y textura, el esmalte puede mostrarse irregular, rugoso, ablandado y con cavitación. La profundidad y causa de estas manchas es el factor clave en la selección y el éxito del tratamiento. El objetivo de este estudio es reportar un caso de reestablecimiento de la estética de la sonrisa en un paciente con hipoplasia de esmalte, a través de la asociación de tratamientos mínimamente invasivos. Para el caso, se optó por la asociación técnica de microabrasión del esmalte dental con ácido fosfórico $37 \%$ asociado a piedra pómez y la macroabrasión del esmalte. Al final del presente caso, la armonía de la sonrisa del paciente ha sido devuelta de forma satisfactoria de acuerdo a sus expectativas. Cuando está indicado, la asociación de tratamientos mínimamente invasivos Cuando está indicado, la asociación de tratamientos mínimamente invasivos para la eliminación de manchas derivadas de la hipoplasia del esmalte demuestra éxito.

Descriptores: Anomalías Dentarias; Esmalte Dental; Estética Dental; Microabrasión del Esmalte; Resinas Compuestas.

\section{INTRODUÇÃO}

A hipoplasia de esmalte pode ser entendida como uma formação incompleta ou defeituosa do esmalte dentário, ou seja, uma deficiência na qualidade do mesmo. Falhas na formação deste substrato podem resultar de manchamento superficial de aspecto esbranquiçado a alterações severas de cor e textura, no qual o esmalte pode se mostrar irregular, rugoso, amolecido e com cavitação ${ }^{1}$. Os sinais encontrados em pacientes acometidos por essa anomalia variam desde sensibilidade dentinária, estética comprometida, facilidade de desenvolvimento de cárie por conta do favorecimento de formação de placa e até problemas de oclusão. Além de problemas psicológicos, causados por conta do comprometimento estética ${ }^{2}$.

A escolha da conduta de tratamento da hipoplasia vai depender da severidade das lesões, que variam de mais brandas, superficiais, até mais críticas ou severas. Para definir a melhor terapia a ser empregada é necessário um bom diagnostico, o qual determinará a profundidade e origem das manchas ${ }^{3}$. Exame clínico minucioso, colhendo todas as características das manchas, é de extrema importância para um plano de tratamento adequado que conduza e um excelente resultado final ${ }^{4}$.
Dentre as terapias, o profissional pode indicar um tratamento menos invasivo como: microabrasão, microabrasao associada ao clareamento dentário ou restaurações adesivas diretas nos casos de manchas superficiais, ou optar por tratamentos mais invasivos como: coroas e facetas, naquelas situações de manchas mais agressivas, onde o esmalte perdeu sua continuidade ${ }^{3}$.

A resina composta é um material restaurador amplamente utilizado e aceito na Odontologia. Com o avanço das tecnologias, existe no mercado uma grande diversidade de materiais restauradores adesivos que proporcionam resultado estético e funcional de excelência, mimetizando a estrutura dentária de forma muito similar à estrutura dentária. Devolvendo a forma, a cor, a opacidade e a textura semelhantes a dentição natural ${ }^{5}$. Possuem uma grande diversidade de cores, texturas e marcas a fim de proporcionar ao profissonal escolhas dependendo de cada caso. É capaz de reanatomizar os dentes de forma simples, com baixo custo e curto intervalo de tempo quando comparado a técnicas indiretas, sendo considerado excelente material de escolha para o reestabelecimento estético de dentes com hipoplasia de esmalte ${ }^{6}$. 
Este relato justifica-se pela importância de se expor um caso clínico de um paciente jovem apresentando hipoplasia de esmalte em dentes anteriores, chegando a comprometer a estética e função. Foi obtido a partir de um planejamento adequado, por meio da anamnese e realização dos exames necessários. Sendo assim, o objetivo do presente estudo foi relatar um caso, apresentando o diagnóstico e tratamento de dentes com hipoplasia de esmalte, com o propósito de melhorar a estética e devolver as suas devidas funções, por meio de procedimentos minimamente invasivos.

\section{CASO CLÍNICO}

Paciente do gênero masculino, 13 anos de idade, compareceu a clínica escola do Centro Universitário Doutor Leão Sampaio acompanhado pela mãe, apresentando lesões de mancha branca no esmalte dentário, dos elementos 11,21,31,32,41 e 42 causando comprometimento da estética e da função destes (Figura 1). Foram realizados procedimentos padrão de anamnese, exames clínicos e radiográficos (Figura 2), para o correto diagnóstico e plano de tratamento, chegando-se ao diagnóstico de hipoplasia de esmalte. $\mathrm{O}$ tratamento proposto foi a microabrasão nas manchas mais superficiais e macroabrasão nas mais profundas, as quais foram identificadas com o auxílio de aparelho fotopolimerizador de lâmpada halógena (Figura 3). Nas manchas mais profundas, que se apresentavam com opacidade exacerbada, devido à hipocalcificação do esmalte, a luz foi barrada. Os dentes com esta alteração apresentam-se com coloração branca e opaca, além de superfície áspera $^{7}$. Previamente ao procedimento de macroabrasão nos elementos da arcada superior, houve a necessidade da confecção de um guia de silicone, devido ao comprometimento do ângulo distoincisal do elemento 11 .

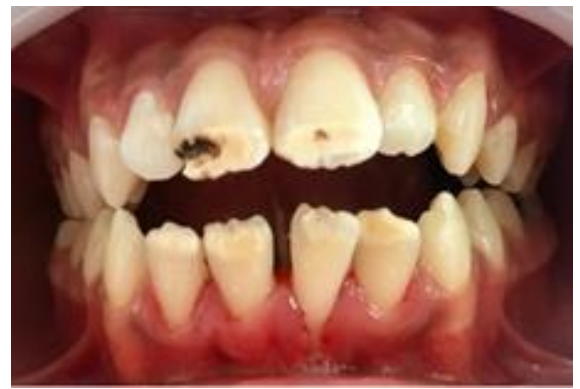

Figura 1: Aspecto inicial das manchas presentes no esmalte vestibular dos dentes 11, 21, 32, 31, 41 e 42.

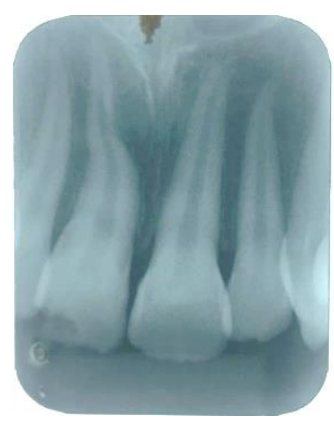

Figura 2: Radiografia periapical dos elementos 11 e 21

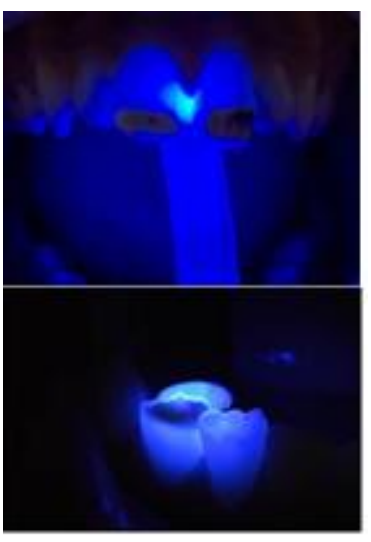

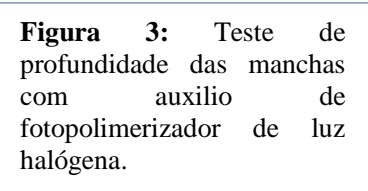

Em seguida procedeu-se à seleção da cor e da resina (A3D-A1E /Resina IPS empress directIvoclar) (Figura 4), isolamento absoluto com dique de borracha e técnica macroabrasiva com o uso da broca carbide esférica $n^{\circ} 6$ removendo a mancha parcialmente (Figuras 5 e 6 ). $\mathrm{O}$ condicionamento ácido, com uso de ácido fosfórico a 37\%, aplicado por 15 segundos em dentina e 30 segundos em esmalte, foi seguido de lavagem abundante por 60 segundos e secagem com papel filtro. Na sequência foi usado o sistema adesivo (Bond 2.1-3M), aplicado com auxílio de microbrush de forma ativa, evaporando o solvente durante 5 segundos por 2 vezes, seguindo a fotoativação por 10 segundos.

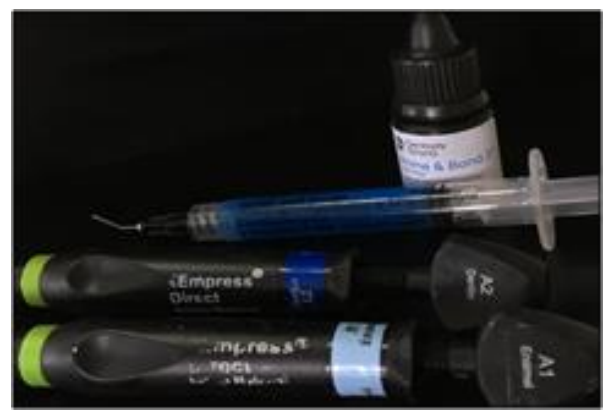

Figura 4: Materiais selecionados.

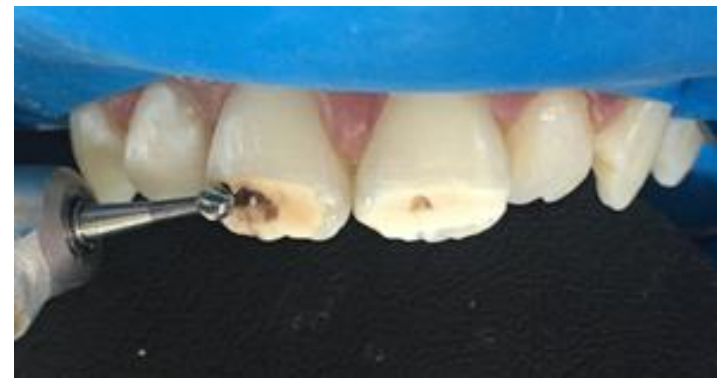

Figura 5: Macroabrasão superficial da mancha com broca carbide esférica $\mathrm{n}^{\circ} 6$.

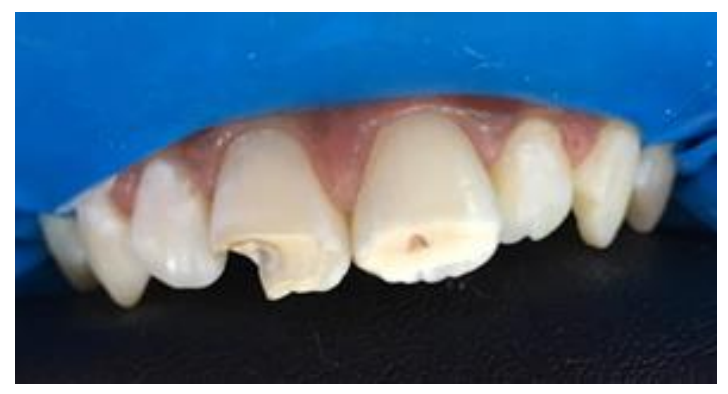

Figura 6: Dente macroabrasionado. 
Concluídas essas etapas, confeccionou-se a restauração, utilizando a guia de silicone como auxilio (Figura 7), iniciando pela face palatina com resina para esmalte (A1E/Resina IPS Empress DirectIvoclar) (Figura 8), seguindo da fotoativação prévia por 15 segundos e remoção desta guia pela face palatina. A resina para dentina (A3D/Resina IPS Empress Direct- Ivoclar) foi depositada de forma incremental, onde uma camada mais opaca foi aplicada inicialmente mascarando áreas com manchamentos e a própria dentina, seguindo de escultura dos mamelos e lóbulos de desenvolvimento, acompanhada da fotoativação. Finalizando a etapa restauradora, aplicou-se resina de esmalte na face vestibular em camada única a fim de minimizar uma possível incorporação de bolhas ${ }^{8}$ (Figura 9).

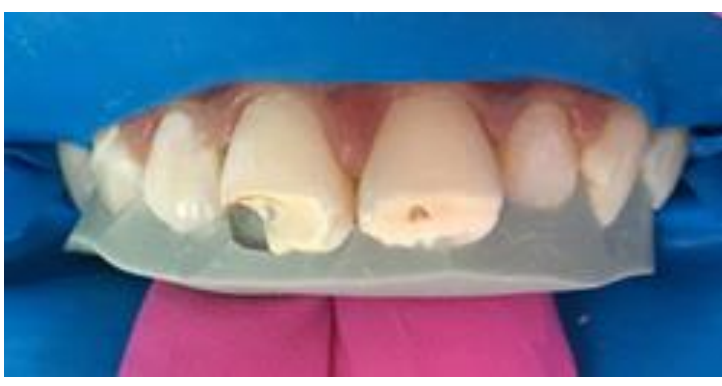

Figura 7: Posicionamento do guia de silicone.

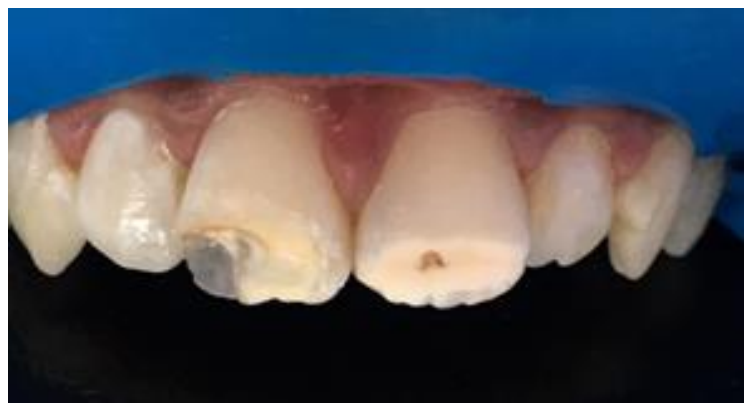

Figura 8: Início da restauração pela face palatina.

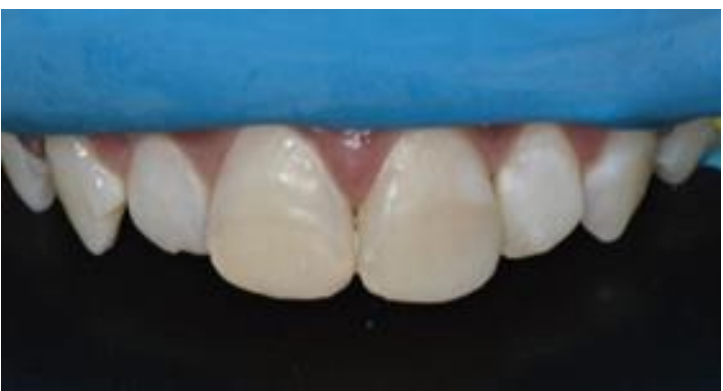

Figura 9: Aspecto final das restaurações antes do acabamento e polimento.

Posteriormente foi realizado o acabamento e polimento, esta etapa têm como finalidade reproduzir características anatômicas, promovendo lisura superficial e brilho além de diminuir a rugosidade. Amenizando a probabilidade de manchamento do material resinoso e o acúmulo de placa, colaborando com a saúde dos tecidos e integridade marginal e estética das restaurações9. O acabamento foi realizado com pontas diamantadas finas (F) e extrafinas (FF) de diferentes formatos tanto na face vestibular quanto na palatina, (Figura 10) assim como os discos soflex (Figura 11) e para polimento da restauração, foram utilizadas pontas abrasivas em formato de disco e chama (Figura 12), seguido o polimento com a escova de carbeto de silício (Figura 13) e com o disco de feltro (Figura 14) e pasta diamantada resultando em brilho $\mathrm{e}$ característica naturais satisfatórias.

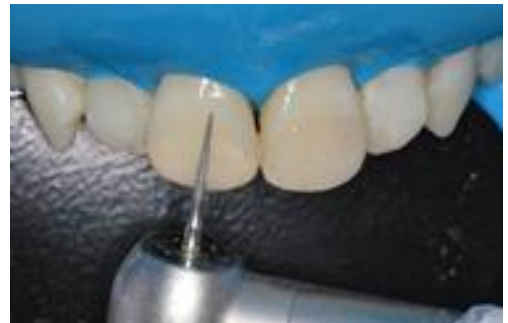

Figura 10: Acabamento das restaurações com broca FF.

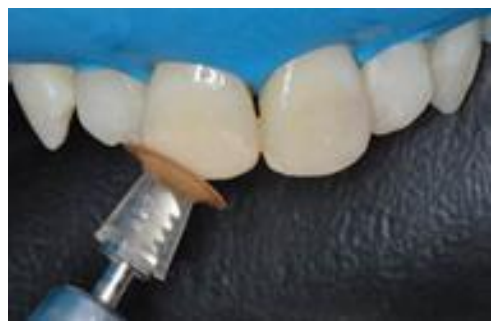

Figura 11: Acabamento com disco Soflex.

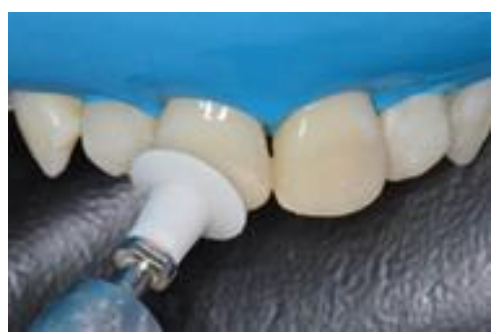

Figura 12: Polimento com ponta abrasiva em forma de disco.

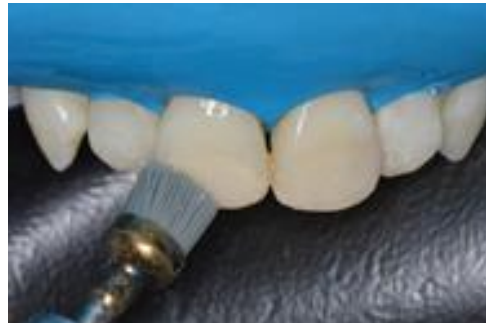

Figura 13: Polimento com escova de carbeto de silício.

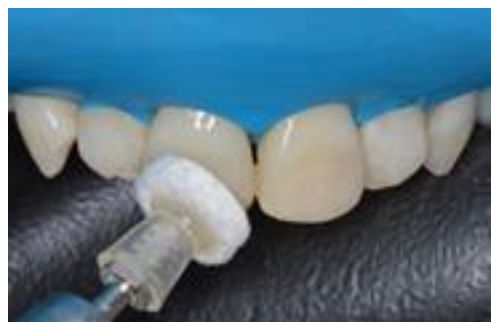

Figura 14: Polimento com disco de feltro e pasta diamantada.

Nos dentes inferiores foi realizado procedimento de microabrasão: antes de serem abrasionados foi realizada profilaxia com pasta de pedra-pomes e água, seguida de isolamento absoluto (Figura 15). Para execução da microabrasão foi aplicado ácido fosfórico a $37 \%$ e pedra-pomes, na proporção de 1:1. Esta pasta foi aplicada na superfície vestibular dos elementos 31, 41, 32 e 42 
com auxilio da taça de borracha em baixa rotação, por 10 segundos (Figura 16). A pasta abrasiva foi lavada com água abundante e o procedimento foi repetido, totalizando 10 aplicações. Após a última lavagem, foi aplicada uma solução de fluoreto de sódio a $2 \%$ neutro com o auxílio de microbrush durante 4 minutos (Figura 17). Esta etapa contribui na formação dos cristais de hidroxiapatita, permitindo a remineralização e diminuição da solubilidade do esmalte $^{10}$.

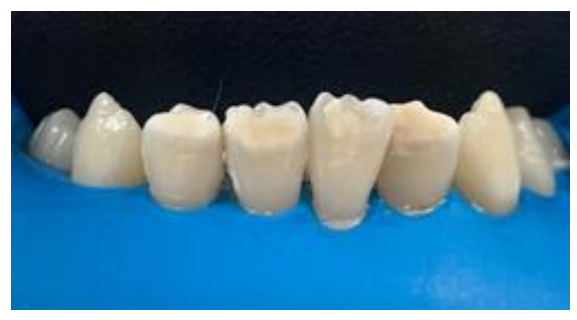

Figura 15: Isolamento absoluto.

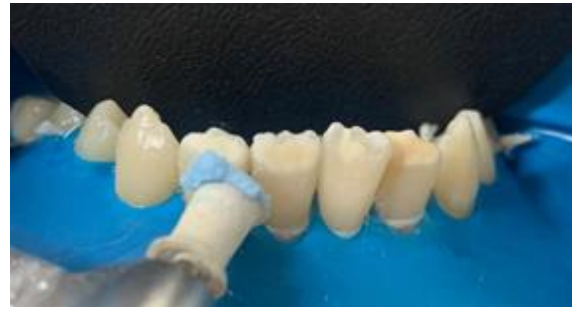

Figura 16: Aplicação de pasta abrasiva com taça de borracha.

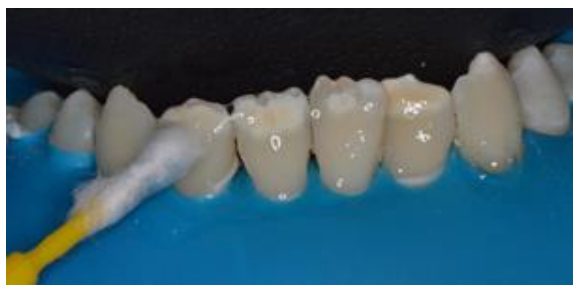

Figura 17: Aplicação tópica de flúor sobre os dentes abrasionados com auxílio de microbrush.

Após verificarmos que a técnica de microabrasão não produziu redução significativa da mancha nos dentes inferiores, optou-se pela técnica macroabrasiva (Figura 18).

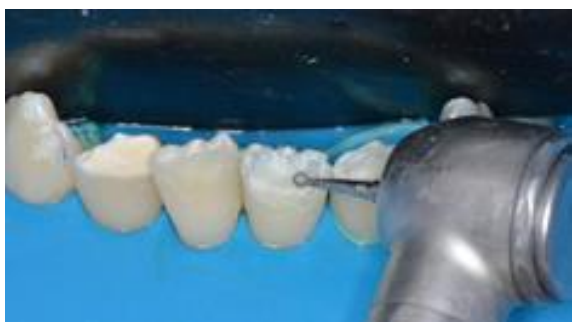

Figura 18: Macroabrasão com ponta diamantada $\mathrm{n}^{\circ} 1012$.

$\mathrm{Na}$ sequência, para a confecção das restaurações foram realizados os mesmos passos dos dentes 11 e 21, sendo realizadas as mesmas etapas; no entanto não houve necessidade da confecção da barreira de silicone, exceto para o elemento 32, pois o procedimento será realizado após a realização do tratamento ortodôntico, devido ao seu posicionamento. Após a finalização da etapa restauradora para os elementos em questão deu-se inicio aos passos de acabamento e polimento
(Figura 19), no qual foi observado o sucesso na remoção das manchas e restabelecimento da harmonia da cor (Figura 20).

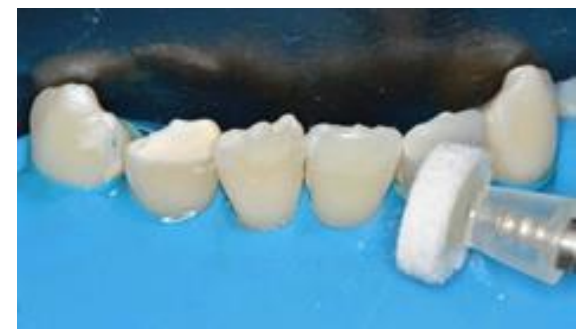

Figura 19: Polimento dos dentes inferiores seguindo os mesmos passos dos dentes superiores.

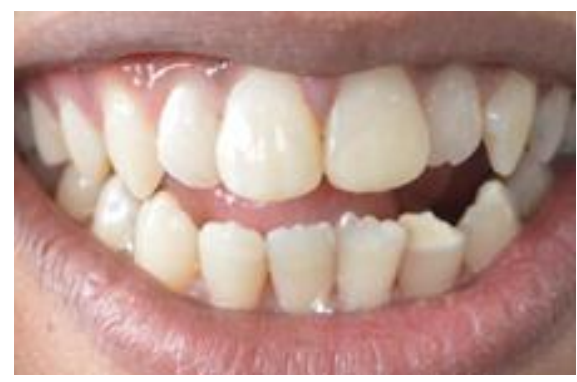

Figura 20: Aspecto final

DISCUSSÃO

Alterações na estrutura do esmalte são descritas como diminuição ou perda local de sua translucidez. Na literatura científica há uma variedade de termos que definem as alterações no esmalte, no entanto existe uma certa dificuldade quanto ao diagnóstico e ao índice utilizado para distinguir cada uma delas ${ }^{11}$.

$\mathrm{Na}$ hipoplasia de esmalte são encontradas pequenas manchas, ranhuras ou fissuras na superfície do tecido, além de irregularidades na espessura, coloração e translucidez, que no caso relatado comprovou-se através do teste realizado com o fotopolimerizador de lâmpada halogena, onde a luz do aparelho foi barrada completamente na região das manchas. Os elementos dentários apresentam-se como pontos ou linhas horizontais, susperfícies rugosas a sondagem e o manchamento geralmente de extensão delimitada, com forma oval ou arredondado em superfícies lisas ${ }^{11,12}$.

No entanto, existem inúmeras alterações que podem estar presentes no esmalte dentário que clinicamente apresentarm-se com as mesmas caracteristicas da hipoplasia, por esse motivo, o diagnóstico deve ser feito de forma clara e detalhada ${ }^{13}$. Como exemplo dessas alterações de esmalte, temos a amelogênese imperfeita e fluorose dentária. A amelogênese imperfeita como um dos diagnósticos diferenciais da hipoplasia, onde sua etiologia está associada a fatores genéticos e pode estar ligada ao taurodontismo, que radiograficamente as câmaras pulpares dos dentes podem apresentar-se aumentadas, diferentemente da hipoplasia de esmalte, e clinicamente pode apresentar-se de três formas: hipoplásica, hipocalficada e hipomaturada. Na forma 
hipoplásica, o esmalte pode conter pouca espessura por conta da formação deficiente da matriz. Já na hipocalcificada, sua calcificação, embora deficiente, é caracterizada por não conter um esmalte resistente, sendo ele opaco e branco amarelado, com estrutura hipomaturada em seus cristais ${ }^{11}$. A fluorose dentária, que também é entendida como um dos diagnósticos diferenciais da hipoplasia de esmalte, é uma anomalia causada pela exposição exagerada a fluoretos durante a fase formativa do tecido. Ela é clinicamente semelhante a hipoplasia. Por isso a importância de se ter uma boa conduta e saber distinguir o diagnóstico, que será determinante para um resultado final satisfatório $^{14}$. É de extrema importância entender e saber diferenciar todas as anomalias que possuem características semelhantes a hipoplasia de esmalte ${ }^{15}$.

O tratamento foi selecionado a partir do grau de profundidade das manchas, no qual o profissional utiliza um aparelho fotopolimerizador de luz halógena, realizando um teste de translucência do esmalte dentário, exame que permite avaliar a propagação da luz sobre a lesão, para identificar a sua profundidade e com isso o nível de comprometimento do esmalte implicando na terapia de escolha ${ }^{16}$.

Entende-se que a técnica de microabrasão do esmalte é um método conservativo e não restaurador, sendo considerada uma técnica segura e minimamente invasiva, age removendo defeitos superficiais de descoloração por meio de aplicações de uma mistura abrasiva onde realmente, uma pequena quantidade, de cerca de $10-30 \mu \mathrm{m}$ do esmalte é removida, após cada aplicação, expondo a camada subsuperficial não manchada, onde a perda de esmalte é clinicamente irreconhecível e insignificante ${ }^{10}$. Essa técnica é mais conservadora e age por meio do uso de diferentes abrasivos associados a soluções químicas, além do ácido fosfórico $37 \%$ e pedra-pomes, mistura que foi utilizada no caso, também pode ser utilizado ácido clorídrico $6 \%$ e carbeto de silício ${ }^{16,17}$. Entretanto, em alguns casos, como o apresentado no presente relato clínico, mostram que certas descolorações são mais resistentes à microabrasão do esmalte, contudo, apresenta limites de indicação, onde não é apropriado na remoção de manchas extensas ou profundas, sendo impossível corrigi-las com tal técnica. Por ser um procedimento pouco invasivo, recomenda-se como primeira alternativa de tratamento para remoção de manchas, porém, caso não seja efetivo, pode-se lançar mão das resinas compostas ${ }^{17}$. Diante de uma odontologia mais conservadora, a microabrasão do esmalte é uma opção viável no tratamento de descolorações no esmalte, mas, em alguns casos, elas podem ser resistentes à técnica ${ }^{10}$, como no presente caso clínico.

No presente relato houve a necessidade da realização da macroabrasão do esmalte, a fim de eliminar o esmalte dental afetado. Portanto utilizou- se pontas diamantadas tornando o procedimento vantajoso, em função de ser pontual e de ser uma superfície de fácil acesso, reduzindo o tempo de trabalho e o estresse do paciente jovem ${ }^{16-18}$. Após a remoção das manchas, foram realizadas as restaurações com resina composta direta, empregando a técnica incremental, onde uma camada mais opaca foi aplicada inicialmente para mascarar qualquer área com manchamento e a própria dentina, pelo motivo de que houve redução da espessura do esmalte.

Como foi observado neste relato é notório que o prognóstico da remoção de manchas por microabrasão do esmalte é totalmente dependente de sua profundidade. Sendo necessário que o profissional esteja preparado para a realização de uma restauração com resina composta, no caso, a macroabrasão ${ }^{17,18}$. Vale ressaltar que o clareamento dental, que é um procedimento padrão quando se fala em estética, não foi a conduta de escolha, pois, além da cor dos dentes não ser a queixa principal do paciente em questão, radiograficamente os elementos dentários encontravam-se em rizogênese incompleta, sendo um fator a ser considerado, além da idade do mesmo.

Nos dias atuais as pessoas estão cada vez mais exigentes em busca da estética do sorriso, com consequente grande procura por tratamentos com esta finalidade, dando prioridade para aqueles procedimentos que tenham bons resultados e que sejam minimamente invasivos. Por esse motivo, as resinas compostas estão no topo dos materiais mais utilizados, estudos são feitos rotineiramente para que seus pontos negativos sejam aprimorados, e ela se torne cada vez mais um material de eleição na odontologia, aliada a uma boa técnica do profissional. Podem ser confeccionadas em uma única sessão clínica, exigem pouco desgaste dental quando comparada a materiais indiretos, propiciam uma boa relação custo/benefício tanto para o profissional quanto para o paciente além de trazerem excelentes resultados ${ }^{18}$

CONCLUSÃO

Quando bem indicados, tratamentos minimamente invasivos ou a associação destes devem ser a primeira escolha para remoção de manchas superficiais, tendo em vista a facilidade da técnica, seu baixo custo quando comparado a procedimentos restauradores indiretos. Resinas compostas de excelência aliadas a uma boa técnica do profissional devolvem a harmonia da cor e da textura, a anatomia detalhada característica de pacientes jovens, a estética e a funcionalidade dos elementos com hipoplasia de esmalte.

\section{REFERÊNCIAS}


1. Oliveira FV, Silva MFA, Nogueira RD, Geraldo Martins VR. Hipoplasia de esmalte em paciente hebiátrico: relato de caso clínico. Rev Odontol Bras Central, 2015;24(68):31-6.

2. Souza BJ, Rodrigues FCP, Lopes GL, Guilherme SA, Freitas CG, Moreira, LCF. Hipoplasia do esmalte: tratamento restaurador estético. Robrac. 2009;18(47):14-9.

3. Catelan A, Pini NIP, Hernandes NMP, Lima DANL, Aguiar FHB. Técnicas minimamente invasivas para estética de manchamentos dentais. Arch Health Invest. 2014;3(4):1-8.

4. Bendo CB, Scarpelli AC, Novaes Junior JB, Vale MPP, Paiva SM, Pordeus IA. Hipoplasia de esmalte em incisivos permanentes: um acompanhamento de 6 meses. RGO. 2017;55(1):108-12.

5. Arruda HS, Leite EBC, Gaspar Junior AA. Reabilitação estética anterior do clareamento á harmonização do sorriso. Odontol.ClinCient.2017;16(2):139-43.

6. Cavalcanti NA, Angolo S, Rodrigues RR. Reanatomização Dental Com Resina Composta. Rev Baiana Odontol. 2014;5(3):182-92.

7. Braga LCC, Mazzetto AH, Grigoletto M, Paganini GA, Vedovello SAS, Jr. Chagas CL. Hipoplasia de esmalte localizada- dente de Turner. RGO. 2005;53(4):329-34.

8. Gouveia CG, Moreira Junior R, Peralta FS, Scherma AP, Resende LM. Facetas diretas de resina composta em dentes anteriores: relato de caso. ClipeOdonto. 2018;9(1):44-50.

9. Menezes MS, Vilela ALR, Silva FP, Reis GR, Borges MG. Acabamento e polimento em resina composta: reprodução natural. Rev Odontol Bras Central. 2014;23(66):124-29.

10. Aranha ACC, Mitsui FHO, Marchi GM. Facetas diretas em resina composta pós-microabrasão relato de caso clínico. J Bras Dent Estet. 2003; 2(5):72-8.

11. Passos IA, Costa JDMC, Melo JM, Forte FDS, Sampaio FC. Defeitos do esmalte: etiologia, características clínicas e diagnóstico. Rev Inst Ciênc Saúde. 2007;25(2):187-92.

12. Sydow DBH, Andrade MRTC, Fidalgo TKS, Valente AR, Tannure PN. Manejo estético da hipoplasia de esmalte em incisivos e caninos permanentes. ClipeOdonto. 2015;7(1):32-6.

13. Hoffmann RHS, Sousa MLR, Cypriano S. Prevalência de defeitos de esmalte e sua relação com cárie dentária nas dentições decídua e permanente. Cad Saúde Pública. 2007;23(2):435-44.

14. Bevilacqua FM, Sacramento T, Felício CM. Amelogênese imperfeita, hipoplasia de esmalte e fluorose dental. UNIARA. 2010;13(2):136-48.

15. Possobon RF, Carrascoza KC, Tomita LM, Ruiz JM, Scarpari CEO, Moraes ABA. Hipoplasia de esmalte em dentes decíduos. RFO UPF. 2006;11(2):73-6.

16. Prado RNS, Zeidan LC, Lotufo MA, Rodrigues JA. Limites da microabrasão do esmalte dental relato de caso. Rev Saúde, 2014;8(1/2):30-6.

17. Silva JMF, Rocha DM, Kimpara ET, Uemura ES. Resinas compostas: estágio atual e perspectivas. Rev Odonto. 2008;16(32):98-104.

18. Souza JB, Rodrigues PCF, Lopes LG, Guilherme AS, Freitas GC, Moreira FCL. Hipoplasia do esmalte: tratamento restaurador estético. ROBRAC. 2009;18(47):14-9.

\section{CONFLITO DE INTERESSES}

Os autores declaram não haver conflitos de interesse.

\section{AUTOR PARA CORRESPONDÊNCIA}

Diala Aretha de Sousa Feitosa

Departamento de Odontologia Centro Universitário Doutor Leão Sampaio Av. Maria Leticia Leite Pereira s/n - Lagoa Seca 63040-405 Juazeiro do Norte - CE, Brasil dialafeitosa@gmail.com
Submetido em 27/07/2020 Aceito em 08/10/2020 